\title{
Assessment of the common carotid artery wall stiffness by Shear Wave Elastography in Behcet's disease
}

\author{
Deniz Alis ${ }^{1}$, Emine Sebnem Memis Durmaz ${ }^{1}$, Ceren Alis², Melih Tutuncu², Sabahattin Saip ${ }^{2}$, \\ Naci Kocer ${ }^{1}$, Civan Islak ${ }^{1}$, Osman Kizilkilic ${ }^{1}$
}

${ }^{1}$ Department of Radiology, ${ }^{2}$ Department of Neurology, Istanbul University, Cerrahpasa Faculty of Medicine, KMPasa, Istanbul, Turkey

\begin{abstract}
Aim: To evaluate endothelial dysfunction and subclinical atherosclerosis in Behcet's disease (BD) by measuring the common carotid artery (CCA) wall stiffness and carotid intima-media thickness (CIMT). Materials and methods: We prospectively evaluated CIMT and the CCA wall stiffness of 34 BD patients and 28 age/sex-matched controls. CIMT measurements were performed from the posterior wall of the carotid artery approximately $10 \mathrm{~mm}$ proximal to the initiation of the carotid bulb using B-mode ultrasound. The stiffness of the CCA was measured from the superficial wall of the CCA using shear wave elastography (SWE). SWE measurements were recorded as shear wave velocity (SWV) using m/s as a unit. Results: The mean right $(0.5 \pm 0.11 \mathrm{~mm})$ and left $(0.5 \pm 0.14 \mathrm{~mm})$ CIMT of the patients were significantly higher compared to the mean right $(0.41 \pm 0.07 \mathrm{~mm})$ and left $(0.41 \pm 0.11 \mathrm{~mm})$ CIMT of the healthy controls $(\mathrm{p}=0.001$ and $\mathrm{p}=0.003$ respectively). The mean right $(3.72 \pm 0.94 \mathrm{~m} / \mathrm{s})$ and left $(3.57 \pm 0.72 \mathrm{~m} / \mathrm{s})$ CCA wall stiffness of the patients were significantly higher compared to the mean right $(2.42 \pm 0.49 \mathrm{~m} / \mathrm{s})$ and left $(2.56 \pm 0.49 \mathrm{~m} / \mathrm{s})$ CCA wall stiffness of the controls $(\mathrm{p}<0.001$ for both). Conclusions: SWE seems to be a promising modality to evaluate endothelial dysfunction in BD by interpreting the arterial stiffness, and SWE might be an important adjunct to clinical and laboratory findings, and imaging modalities to assess cardiovascular risk in BD. Moreover, SWE evaluation of the arterial stiffness might assist us to understand pathophysiological aspects of BD.
\end{abstract}

Keywords: arterial stiffness; Behcet's disease; intima-media thickness; neuro-Behcet's disease, SWE

\section{Introduction}

Behcet's disease (BD) is an inflammatory disease with unknown origin, first described by the Turkish dermatologist, Hulusi Behcet [1]. BD characterized by recurrent oral aphthous lesions, genital ulcerations and uveitis and might also present with cardiovascular, nervous, gastrointestinal and urogenital manifestations. The prevalence of the cardiovascular involvement in BD has been documented up to $40 \%$ [2]. Many cases present vas-

Received 01.05.2018 Accepted 13.09.2018

Med Ultrason

2018, Vol. 20, No 4, 446-452

Corresponding author: Deniz Alis, M.D.

Istanbul University, Cerrahpasa Faculty of

Medicine, Department of Radiology, KMPasa

34098 Istanbul, Turkey

E-mail: drdenizalis@gmail.com cular complications, venous (14-40\% of cases) and/or arterial $(0.5 \%-17 \%$ of cases $)$ [2]. The association between endothelial injury and atherosclerosis, a chronic inflammatory condition characterized by the changes in the arterial wall, has been well established $[3,4]$. Although exact mechanisms that cause arterial involvement is not clearly understood, many studies have proposed that endothelial dysfunction (ED) secondary to vasculitis might play a vital role in the development of the cardiovascular manifestations of BD and many other systemic inflammatory diseases as well [5-9]. Since early diagnosis of the arterial changes is crucial to prevent cardiovascular complications, different diagnostic methods, including flow-mediated dilatation (FMD), carotid intima-media thickness (CIMT), ankle-brachial pressure index (ABPI) and pulse wave velocity (PWV) measurements have been investigated by many researchers to detect subclinical 
atherosclerosis in BD $[10,11]$. Despite many promising results regarding these methods in the diagnosis of subclinical atherosclerosis, controversial results have also been reported [12-15].

Shear wave elastography (SWE) is a novel elastographic method, which quantifies the elasticity of structures by tracking shear waves that pass through tissues [16]. SWE differs from strain elastography by its ability to yield quantitative and less-operator dependent information regarding tissue stiffness. The feasibility and effectiveness of SWE in the diagnosis of various pathologies of many organs have been demonstrated including liver, breast, nerves, thyroid, testes, and soft tissue lesions [16-20]. Recently, vascular applications of SWE have been a topic of interest. Studies with promising results have been reported regarding the utilization of SWE in the evaluation of vessels [21-26].

In this study, we evaluated the common carotid artery (CCA) stiffness in BD patients with SWE in order to assess whether SWE might be a feasible and efficacious tool to detect early changes in the arterial wall in BD.

\section{Materials and methods}

The local Ethics Committee approved this prospective study, which was conducted between August 2017 and February 2018. This study was carried out according to the ethical principles of the Declaration of Helsinki. Informed consents were obtained from all patients. Patients with BD who fulfilled the international Behcet study group criteria [27] and age/sex-matched healthy controls were included in the study. Exclusion criteria were the followings: presence of diabetes mellitus; history of stroke or myocardial infarct; current or former smoking; presence of hypertension (defined as systolic blood pressure $>140 \mathrm{~mm} \mathrm{Hg}$ and diastolic blood pressure $>90 \mathrm{~mm} \mathrm{Hg}$ ); obesity (defined as BMI $>30 \mathrm{~kg} / \mathrm{m}^{2}$ ); pregnancy; using hormone replacement drugs, lipid-lowering drugs, antihypertensive drugs and steroids; presence of renal or liver disease; presence of autoimmune disease rather than $\mathrm{BD}$; having abnormal lipid profiles (defined as total cholesterol or triglyceride levels in fasting plas$\mathrm{ma}>240 \mathrm{mg} / \mathrm{dl}$ and $>160 \mathrm{mg} / \mathrm{dl}$, respectively); having cardiovascular involvement of BD (expect vascular involvement in central nervous system). Additionally, all BD patients should have an active disease to be included in the study. Active disease was defined as having two or more of the following symptoms or manifestations: oral aphthous ulcers, genital ulcers, skin lesions, eye involvement, neurological involvement and involvement of other systems. The healthy control group also had the same exclusion criteria with BD patients. To perform subgroup analysis, BD patients were subgrouped as patients with central nervous system involvement, namely NeuroBehcet's disease (NBD), and patients without central nervous system involvement. Using standard laboratory methods, C-reactive protein (CRP) was measured from fasting blood samples of all subjects.

\section{CIMT and SWE measurements}

A single radiologist performed all CIMT and SWE measurements (E.S.M.D, 5 years of ultrasound and SWE experience). Patients were lying in a comfortable supine position during the examination and were asked to slightly extend and turn their head to the contralateral side of

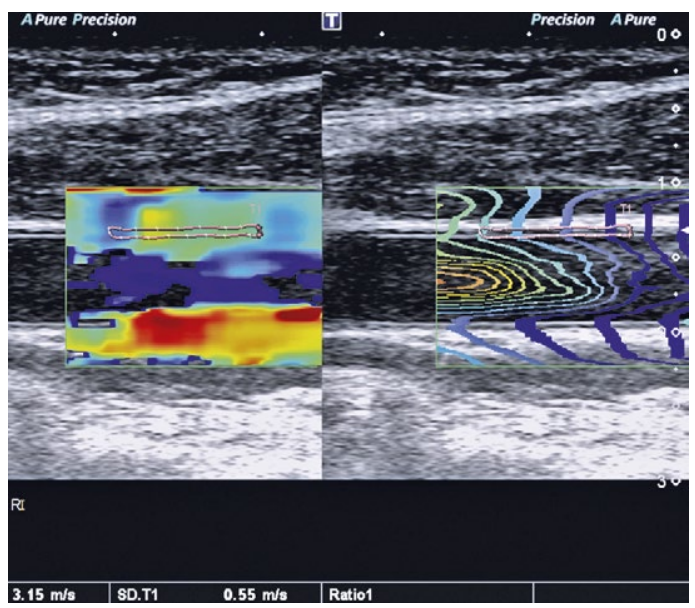

Fig 1. SWE examination of a 40-year-old female with known BD. The observer carefully drew ROI onto the superficial wall of the CCA ensuring not to include vessel lumen or surrounding tissue within ROI. Also note the shear wave mapping inside the vessel is an artifact caused by reverberation from the vessel wall and layers structure.

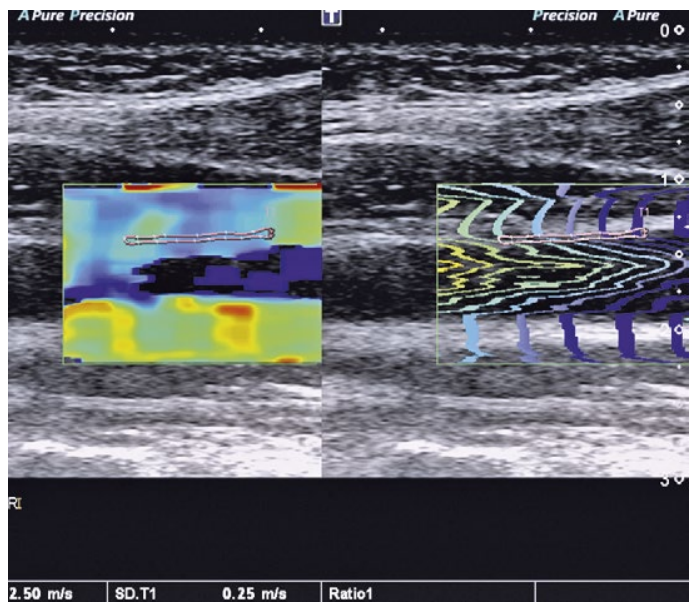

Fig 2. Carotid SWE image of a 38-year-old healthy female shows lower stiffness value compared to the patient with $\mathrm{BD}$ in figure 1 . 
the examined CCA. All examinations were performed with the same ultrasound unit (Aplio 500; Toshiba Medical Systems Co, Ltd, Tokyo, Japan) with a SWE application using a high-frequency linear transducer (7-14 $\mathrm{MHz}$ ). First, longitudinal images of the carotid system were obtained at the late diastolic phase. CIMT measurements were performed from the posterior wall of the CCA approximately $10 \mathrm{~mm}$ proximal to the initiation of the carotid bulb. The zoom levels were adjusted according to the comfort of the operator during examinations. Subsequently, the same operator performed SWE examination of the CCA at the same session. The SWE examinations were performed from the superficial wall of the CCA within $1-4 \mathrm{~cm}$ proximal to the carotid division. First, the observer captured longitudinal images of the CCA at the end of diastole. Second, the observer placed a q-Box over the CCA and then manually drew a region of interest (ROI) onto the anterior wall of the CCA. The ROIs were precisely drawn onto the carotid wall avoiding vessel lumen or surrounding tissue. The operator performed five valid measurements for each CCA, and the average values of these measurements were accepted as the mean value. All of the SWE measurements were recorded as shear wave velocities (SWV) using $\mathrm{m} / \mathrm{s}$ as unit. All data were recorded in our local picture archiving and communication system (Extremepacs, Ankara, Turkey). Figure 1 demonstrates a SWE measurement of a patient with $\mathrm{BD}$ and figure 2 demonstrates a SWE measurement of a healthy control.

\section{Statistical analyses}

Statistical analyses were performed using the SPSS software version 21 . The variables were investigated using visual (probability plots and histograms) and analytical methods (Kolgomorov-Smirnov/Shapiro-Wilk's test) to detect whether or not they were normally distributed. Descriptive analyses were presented using means and standard deviations for the numerical variables. Since the measured CIMT and SWE values were not normally distributed, non-parametric tests were conducted to compare these parameters. The Chi-square test or Fisher's exact test (when chi-square test assumptions do not hold due to low expected cell counts) was used to compare categorical variables. While investigating the associations between non-normally distributed variables, the correlation coefficients and their significance were calculated using the Spearman test. A p value of less than 0.05 was considered to show a statistically significant result

\section{Results}

Finally, a total of 34 patients and 28 healthy controls were enrolled in the study. No significant differences were observed between the two groups regarding age, sex, BMI, diastolic and systolic blood pressure. The mean CRP value of the patients $(9.36 \pm 2.32 \mathrm{mg} / \mathrm{l})$ was significantly higher than the mean CRP value of the healthy controls $(0.6 \pm 0.36 \mathrm{mg} / \mathrm{l})(\mathrm{p}<0.001)$. The mean right $(0.5 \pm 0.11 \mathrm{~mm})$ and left $(0.5 \pm 0.14 \mathrm{~mm})$ CIMT of the patients were significantly higher compared to the mean right $(0.41 \pm 0.07 \mathrm{~mm})$ and left $(0.41 \pm 0.11 \mathrm{~mm})$ CIMT of the healthy controls $(\mathrm{p}=0.001$ and $\mathrm{p}=0.003$ respectively). The mean right $(3.72 \pm 0.94 \mathrm{~m} / \mathrm{s})$ and left $(3.57 \pm 0.72$ $\mathrm{m} / \mathrm{s}$ ) CCA wall stiffness of the patients were significantly higher compared to the mean right $(2.42 \pm 0.49 \mathrm{~m} / \mathrm{s})$ and left $(2.56 \pm 0.49 \mathrm{~m} / \mathrm{s})$ CCA wall stiffness of the controls $(p<0.001$ for both). Table I demonstrates the demographics, clinical, CIMT and carotid SWE findings of patients with BD and healthy controls and the results of univariable analyses. No correlation was observed between the left CCA stiffness and CIMT ( $\mathrm{p}=0.12$ for patients and

Table I. Baseline characteristics, Shear Wave Elastography and carotid intima-media thickness parameters of patients with Behcet's disease and control group

\begin{tabular}{lllc}
\hline & Behcet's Disease & Healthy controls & Univariatep-value \\
\hline Age & $40.38 \pm 10.39$ & $36.28 \pm 8.26$ & 0.064 \\
Sex (M:F) & $18: 16$ & $15: 13$ & 0.36 \\
BMI (kg/m ${ }^{2}$ ) & $25.29 \pm 1.84$ & $24.44 \pm 1.38$ & 0.24 \\
Systolic blood pressure (mmHg) & $129.76 \pm 7.31$ & $127.67 \pm 6.70$ & 0.11 \\
Diastolic blood pressure (mmHg) & $77.47 \pm 6.65$ & $75.10 \pm 5.8$ & 0.094 \\
CRP (mg/l) & $9.36 \pm 2.32$ & $0.6 \pm 0.36$ & $<0.001$ \\
Right CIMT (mm) & $0.5 \pm 0.11$ & $0.41 \pm 0.07$ & 0.001 \\
Left CIMT (mm) & $0.5 \pm 0.14$ & $0.41 \pm 0.11$ & 0.003 \\
Right CCA stiffness (m/s) & $3.72 \pm 0.94$ & $2.42 \pm 0.49$ & $<0.001$ \\
Left CCA stiffness (m/s) & $3.57 \pm 0.72$ & $2.56 \pm 0.49$ & $<0.001$ \\
\hline
\end{tabular}

*All values are expressed as mean \pm SD. M - male; F - female; SWE - Shear Wave Elastography; CIMT - carotid intima-media thickness; $\mathrm{CRP}$ - C-reactive protein: CCA - common carotid artery; BMI - body mass index 
Table II. Baseline characteristics, Shear Wave Elastography and carotid intima-media thickness parameters of Behcet's Disease patients with and without neurological involvement.

\begin{tabular}{llll}
\hline & Behcet's Disease & Neurobehcet's Disease & Univariate p-value \\
\hline Age & $41.23 \pm 10.27$ & $39.33 \pm 12.83$ & 0.69 \\
BMI $\left(\mathrm{kg} / \mathrm{m}^{2}\right)$ & $25.42 \pm 1.39$ & $25.14 \pm 1.77$ & 0.10 \\
Duration of the disease (years) & 7.758 (range 2-22) & 10.73 (range 2-38) & 0.7 \\
Systolic blood pressure (mm Hg) & $130.36 \pm 6.43$ & $129 \pm 8.47$ & 0.82 \\
Diastolic blood pressure (mm Hg) & $77.36 \pm 6.43$ & $75.10 \pm 5.8$ & 0.74 \\
CRP (mg/l) & $9.32 \pm 2.35$ & $9.40 \pm 2.37$ & 0.91 \\
Right CIMT (mm) & $0.48 \pm 0.10$ & $0.53 \pm 0.12$ & 0.25 \\
Left CIMT (mm) & $0.48 \pm 0.11$ & $0.54 \pm 0.17$ & 0.47 \\
Right CCA stiffness (m/s) & $3.62 \pm 1$ & $3.85 \pm 0.86$ & 0.41 \\
Left CCA stiffness (m/s) & $3.53 \pm 0.85$ & $3.62 \pm 0.54$ & 0.47 \\
\hline
\end{tabular}

*All values are expressed as mean \pm SD; SWE - Shear Wave Elastography; CIMT - carotid intima-media thickness; CRP - C-reactive protein: CCA - common carotid artery; BMI - body mass index

$\mathrm{p}=0.24$ for controls) and the right CCA stiffness and CIMT ( $\mathrm{p}=0.79$ for patients and $\mathrm{p}=0.97$ for controls) measurements of patients and controls.

Among 34 patients, 15 were classified as NBD given the presence of central nervous system manifestations (13 patients had parenchymal and 2 patients had vascular involvement). When we compared $\mathrm{BD}$ patients without the neurological involvement and NBD patients no significant difference was observed between two groups regarding age, CRP, BMI, duration of the disease, diastolic and systolic blood pressure. Moreover, no significant difference was observed between two groups regarding right CIMT (BD $=0.48 \pm 0.10 \mathrm{~mm}, \mathrm{NBD}=0.53 \pm 0.12 \mathrm{~mm})$, right CCA wall stiffness $(B D=3.62 \pm 1 \mathrm{~m} / \mathrm{s}, \mathrm{NBD}=3.85 \pm 0.86$ $\mathrm{m} / \mathrm{s})$, left CIMT $(\mathrm{BD}=0.48 \pm 0.11 \mathrm{~mm}, \mathrm{NBD}=0.54 \pm 0.17$ $\mathrm{mm})$ and left CCA stiffness $(B D=3.53 \pm 0.85 \mathrm{~m} / \mathrm{s}$, $\mathrm{NBD}=3.62 \pm 0.54 \mathrm{~m} / \mathrm{s})(\mathrm{p}>0.05)$. In table II are detailed the demographics, clinical, CIMT and carotid SWE findings of patients with $\mathrm{BD}$ and $\mathrm{NBD}$ and results of univariable analyses as well.

\section{Discussions}

We found that both CIMT and the CCA wall stiffness were higher in $\mathrm{BD}$ compared to healthy controls. However, no correlation was observed between the CIMT and stiffness values. We also found no significant difference between patients with NBD and BD regarding the CIMT and the CCA wall stiffness.

Cardiovascular complications, especially complications secondary to the arterial involvement, in BD cause significant mortality and morbidity; thus, determination of the cardiovascular involvement at the preclinical stage has great importance for the prognosis. Besides imaging modalities, many markers have been investigated to detect $\mathrm{BD}$ patients with increased susceptibility to future cardiovascular complications. Chambers et al [28] demonstrated the role of oxidative stress in the pathophysiology of BD by showing decreased levels of nitric oxide in BD patients. Moreover, further studies reported increased levels of thrombomodulin, CRP, homocysteine, von Willebrand factor, thromboxane, oxygen-derived free radicals by neutrophils or asymmetric dimethylarginine [28-34]. All aforementioned markers suggested the presence of endothelial dysfunctions and inflammation in $\mathrm{BD}$, which might potentially enhance atherosclerosis and increase the arterial stiffness.

The association between increased arterial stiffness and development of atherosclerosis has been well established [35,36]. Increased arterial stiffness has been reported in various conditions including hypertension, stroke and heart failure $[21,35,36]$. Recently, the arterial stiffness in $\mathrm{BD}$ has become a favorite topic for analyses and many studies have been conducted to predict early ED using different methods including PWV, FMD, ABPI [10,11,37]. Reduced FMD and increased PWV values, which reflect increased arterial stiffness, in BD have been documented by many studies $[10,14,37,38]$. We also found increased arterial stiffness in BD patients compared to healthy controls. However, some authors observed no difference between BD and healthy controls regarding arterial stiffness $[13,15,16]$. Yolbas et al [15] asserted that BD dominantly affects the venous system rather than the arteries and claimed that one might expect normal arterial stiffness indexes and CIMT values in BD since both parameters reflect arterial function and structure.

Increased CIMT is a well-known and accepted surrogate marker for early atherosclerosis and ED as well [39-41]. Many authors have investigated CIMT of BD patients and reported increased CIMT $[25,42,43]$. We also found increased CIMT in BD compared to healthy 
controls. Contrary to aforementioned studies and our result, Rhee et al [44] and Seyahi et al [45] reported no difference between $\mathrm{BD}$ and healthy controls regarding CIMT. Additionally, Caldas et al [14] and Yolbas et al [15] also reported no difference between BD and healthy controls regarding CIMT. Caldas et al [14] claimed that the increased CIMT in BD patients reported in previous studies might have emerged due to the presence of traditional risk factors. Yolbas et al [15] contended that changes in the arterial wall might only be limited to the involved regions in $\mathrm{BD}$.

Regardless of the contrary results, the histopathological studies that evaluated the wall of the aorta and carotid artery in BD strongly support our findings. Intense infiltration of the media by inflammatory cells, loss of elastic fibers and proliferation of fibroblasts in the media were documented in the active stage, and fibrous thickening of the intima and adventitia in the chronic stage were observed in these studies [46,47]. It has been well established that increased fibroblast and fibrosis positively correlated with the stiffness values. Moreover, these changes could also explain the increased thickening of the arterial wall. Therefore, as we documented in our study, we suggest that BD patients have increased arterial stiffness and CIMT values, which might reflect the cardiovascular risk in $\mathrm{BD}$.

In contrast to techniques including FMD, ABPI and PWV that measure the circumferential elastic modulus of the arterial wall, the SWE assesses the longitudinal elastic modulus [22]. Thus, SWE allows operators to precisely obtain topographical description both in space and time of the arterial stiffness of the segment being studied; hence, it might yield superior information regarding viscoelastic properties of the arterial wall [22].

Our study group also composed of a considerable amount of BD patients with neurologic involvement, namely NBD. Two types of NBD have been described: parenchymal form, commonly involving brainstem, cerebral white matter and basal ganglia; and vascular form, involving the intracranial arteries and veins [48]. Recent radiological and histological studies have been demonstrated that the inflammation of the small veins is the main mechanism behind the parenchymal lesions in NBD $[49,50]$. Besides, studies that evaluated vertebral arteries with Doppler ultrasound and magnetic resonance angiography reported significant difference regarding flow pattern and diameter of vertebral arteries between $\mathrm{BD}$ and NBD patients and claimed that these changes might be associated with the inflammatory changes in the brain parenchyma [51,52]. We compared the CCA wall stiffness and CIMT of the BD and NBD patients, yet we found no significant difference.
We had several limitations in our study. First, we had a relatively small number of participants in our study. Second, we did not calculate the disease severity score of the patients; hence, we did not evaluate the correlation between the arterial stiffness and disease severity scores of the patients. Third, we did not compare SWE with other methods including FMD and PWV. Therefore, we suggest that a comparative study of SWE, PWV and FMD in the evaluation of arterial stiffness in BD should be carried out in the future. Fourth, treatment status and regimens of the patient were not taken into account, which might affect CIMT and the CCA stiffness. Lack of interobserver variability calculation since only one observer performed all examinations, and also lack of intraobserver variability assessments should be also acknowledged as other limitations of the study. Finally, we only evaluated stiffness of the carotid artery; thus, we suggest that future studies that measure the stiffness of different arteries, including the brachial and femoral artery, in $\mathrm{BD}$ should be conducted.

In conclusion, we evaluated the CCA wall stiffness of BD patients for the first time using SWE. We highlight that SWE seems to be a promising modality to assess endothelial dysfunction in $\mathrm{BD}$ by interpreting the arterial stiffness and SWE might be an important adjunct to clinical and laboratory findings and imaging modalities to evaluate cardiovascular risk in BD. We also emphasize that SWE evaluation of the arterial stiffness might assistus to understand the pathophysiological aspects of $\mathrm{BD}$.

\section{Conflict of interest: none}

\section{References}

1. International Team for the Revision of the International Criteria for Behçet's Disease (ITR-ICBD). The International Criteria for Behçet's Disease (ICBD): a collaborative study of 27 countries on the sensitivity and specificity of the new criteria. J Eur Acad Dermatol Venereol 2014;28:338-347.

2. Desbois AC, Wechsler B, Cluzel P, et al. Cardiovascular involvement in Behcet's disease. Rev Med Interne 2014;35:103-111.

3. Libby P. Inflammation in atherosclerosis. Nature 2002;420:868-874.

4. Celermajer DS, Sorensen KE, Gooch VM, et al. Non-invasive detection of endothelial dysfunction in children and adults at risk of atherosclerosis. Lancet 1992;340:1111-1115.

5. Schmitz-Huebner U, Knop J. Evidence for an endothelial dysfunction in association with Behcet's disease. Thromb Res 1984;34:277-285.

6. Haznedaroglu IC, Ozcebe OI, Ozdemir O, Celik I, Dündar SV, Kirazli S. Impaired haemostatic kinetics and 
endothelial function in Behcet's disease. J Intern Med 1996;240:181-187.

7. Kiraz S, Ertenli I, Ozturk MA, Haznedaroğlu IC, Celik I, Calgüneri M. Pathological haemostasis and 'prothrombotic state' in Behcet's disease. Thromb Res 2002;105:125133.

8. Acikgoz N, Ermiş N, Yağmur J, et al. Elevated oxidative stress markers and its relationship with endothelial dysfunction in Behçet disease. Angiology 2011;62:296-300.

9. Onur E, Kabaroglu C, Inanir I, et al. Oxidative stress impairs endothelial nitric oxide levels in Behcets' disease. $\mathrm{Cu}$ tan Ocul Toxicol 2011;30:217-220.

10. Merashli M, Ster IC, Ames PR. Subclinical atherosclerosis in Behcet's disease: A systematic review and meta-analysis. Semin Arthritis Rheum 2016;45:502-510.

11. Uyar B, Solak A, Genç B, et al. Evaluation of Arterial Stiffness in Patients with Behçet's Disease by Using Noninvasive Radiological Methods such as Intima-Media Thickness of the Carotid, Ankle-Brachial Pressure Index, Coronary Artery Calcium Scoring, and Their Relation to Serum Fetuin-A levels: A Case-Control Study. Ann Dermatol 2015;27:702-708.

12. Kurum T, Yildiz M, Soy M, Ozbay G, Alimgil L, Tüzün B. Arterial distensibility as determined by carotid-femoral pulse wave velocity in patients with Behcet's disease. Clin Rheumatol 2005;24:134-138.

13. Alan S, Ulgen MS, Akdeniz S, Alan B, Toprak N. Intima media thickness and arterial distensibility in Behçet's disease. Angiology 2004;55:413-419.

14. Caldas CA, Borba EF, Bortolotto LA, Medeiros DM, Bonfa E, Gonçalves CR. Increased arterial stiffness assessed by pulse wave velocity in Behçet's disease and its association with the lipid profile. J Eur Acad Dermatol Venereol 2013;27:454-459.

15. Yolbas S, Gözel N, Dağlı MN, Koca SS, Dönder E. Carotid artery stiffness in Behçet's disease. Eur J Rheumatol 2017;4:122-126.

16. Hekimoglu K, Yildrim Donmez F, Arslan S, Ozdemir A, Demir C, Yazici C. The role of shear wave elastography in the diagnosis of chronic autoimmune thyroiditis. Med Ultrason 2015; 17:322-326.

17. Guibal A, Renosi G, Rode A, et al. Shear wave elastography: An accurate technique to stage liver fibrosis in chronic liver diseases. Diagn Interv Imaging 2016;97:91-99.

18. Samir AE, Allegretti AS, Zhu Q, et al. Shear wave elastography in chronic kidney disease: a pilot experience in native kidneys., BMC Nephrol 2015;16:119.

19. Dikici AS, Er ME, Alis D, et al. Is There Any Difference Between Seminomas and Nonseminomatous Germ Cell Tumors on Shear Wave Elastography? A Preliminary Study. J Ultrasound Med 2016;35:2575-2580.

20. Kantarci F, Ustabasioglu FE, Delil S, et al. Median nerve stiffness measurement by shear wave elastography: a potential sonographic method in the diagnosis of carpal tunnel syndrome. Eur Radiol 2014;24:434-440.

21. Li Z, Du L, Wang F, Luo X. Assessment of the arterial stiffness in patients with acute ischemic stroke using longitudi- nal elasticity modulus measurements obtained with Shear Wave Elastography. Med Ultrason 2016;18:182-189.

22. Messas E, Pernot M, Couade M. Arterial wall elasticity: state of the art and future prospects. Diagn Interv Imaging 2013;94:561-569.

23. Caenen A, Shcherbakova D, Verhegghe B, et al. A versatile and experimentally validated finite element model to assess the accuracy of shear wave elastography in a bounded viscoelastic medium. IEEE Trans Ultrason Ferroelectr Freq Control 2015;62:439-450.

24. Couade M, Pernot M, Prada C, et al. Quantitative assessment of arterial wall biomechanical properties using shear wave imaging. Ultrasound Med Biol 2010;36:1662-1676.

25. Dumont DM, Doherty JR, Trahey GE. Noninvasive assessment of wall-shear rate and vascular elasticity using combined ARFI/SWEI/spectral Doppler imaging system. Ultrason Imaging 2011;33:165-188.

26. Gulsen F, Samanci C, Memis Durmaz ES, et al. Brachial Artery Wall Stiffness Assessment by Shear Wave Elastography: A Promising New Diagnostic Tool for Endothelial Dysfunction Detection. J Ultrasound Med 2018;37:1977-1983.

27. Criteria for diagnosis of Behçet's disease. International Study Group for Behçet's Disease. Lancet 1990;335:10781080.

28. Chambers JC, Haskard DO, Kooner JS. Vascular endothelial function and oxidative stress mechanisms in patients with Behçet's syndrome. J Am Coll Cardiol 2001;37:517520 .

29. Sandikci R, Turkmen S, Guvenen G, et al. Lipid peroxidation and antioxidant defence system in patients with active or inactive Behcet's disease. Acta Derm Venereol 2003;83:342-346.

30. Ekiz O, Balta I, Sen BB, et al. Mean platelet volume in recurrent aphthous stomatitis and Behcet disease. Angiology 2014;65:161-165.

31. Haznedaroglu IC, Ozdemir O, Ozcebe O, Dündar SV, Kirazli S. Circulating thrombomodulin as a clue of endothelial damage in Behcet's disease. Thromb Haemost 1996;75:974-975.

32. Ozoran K, Dugun H, Gurler A, Tutkak H, Tokgöz G. Plasma von Willebrand factor, tissue plasminogen activator, plasminogen activator inhibitor, and antithrombin III levels in Behcet's disease. Scand J Rheumatol 1995;24:376-382.

33. Mege JL, Dilsen N, Sanguedolce V, et al. Overproduction of monocyte derived tumor necrosis factor alpha, interleukin (IL) 6, IL-8 and increased neutrophil superoxide generation in Behcet's disease. A comparative study with familial Mediterranean fever and healthy subjects. J Rheumatol 1993;20:1544-1549.

34. Yuksel M, Yildiz A, Oylumlu M, et al. Novel markers of endothelial dysfunction and inflammation in Behçet's disease patients with ocular involvement: epicardial fat thickness, carotid intima media thickness, serum ADMA level, and neutrophil-to-lymphocyte ratio. Clin Rheumatol 2016;35:701-708.

35. Vlachopoulos C, Aznaouridis K, Stefanadis C. Prediction of cardiovascular events and all-cause mortality with arte- 
rial stiffness: A systematic review and meta-analysis. J Am Coll Cardiol 2010;55:1318-1327.

36. Quinn U, Tomlinson LA, Cockcroft JR. Arterial stiffness. JRSM Cardiovasc Dis 2012;1:cvd.2012.012024. doi:10.1258/cvd.2012.012024

37. Yilmaz S, Celik G, Esmen SE. Assessment of arterial stiffness in patients with inactive and active Behçet's disease. Scand J Rheumatol 2014;43:63-69.

38. Ozuguz P, Karabulut AA, Tulmac M, Kisa U, Kocak M, Gunduz O. Markers of endothelial dysfunction and evaluation of vascular reactivity tests in Behcet disease. Angiology 2014;65:937-943.

39. Touboul PJ, Hennerici MG, Meairs S, et al. Mannheim intima-media thickness consensus. Cerebrovasc Dis 2004; 18:346-349.

40. Bots ML, Dijk JM, Oren A, Grobbee DE. Carotid intimamedia thickness, arterial stiffness and risk of cardiovascular disease: current evidence. J Hypertens 2002;20:2317-2325.

41. Bots ML, Hoes AW, Koudstaal PJ, Hofman A, Grobbee DE. Common carotid intima-media thickness and risk of stroke and myocardial infarction: the Rotterdam Study. Circulation 1997;96:1432-1437.

42. Hong SN, Park JC, Yoon NS, et al. Carotid artery intima-media thickness in Behcet's disease patients without significant cardiovascular involvement. Korean J Intern Med 2008;23:87-93.

43. Yıldırım A, Karakas MS, Kılınc AY, Altekin RE, Yalçınkaya AS. Evaluation of arterial stiffness and subclinical atherosclerosis in patients with Behçet's disease without cardiovascular involvement. Turk Kardiyol Dern Ars 2016;44:575-581.
44. Rhee MY, Chang HK, Kim SK. Intima-media thickness and arterial stiffness of carotid artery in Korean patients with Behçet's disease. J Korean Med Sci 2007;22:387-392.

45. Seyahi E, Ugurlu S, Cumali R, et al. Atherosclerosis in Behçet's Syndrome. Semin Arthritis Rheum 2008;38:1-12.

46. Matsumoto T, Uekusa T, Fukuda Y. Vasculo-Behçet's disease: a pathologic study of eight cases. Hum Pathol 1991;22:45-51.

47. Urayama A, Sakuragi S. Sakai F, Tanaka Y, Koseki T. Angio-Behcet's syndrome. In: Inaba G. (Ed.). International Symposium on Behcet's Disease. Tokyo. Japan, University of Tokyo Press. 1982:17 1- I76.

48. Mohammed RH, Nasef A, Kewan HH, Al Shaar M. Vascular neurobehcet disease: correlation with current disease activity forum and systemic vascular involvement. Clin Rheumatol 2012;31:1033-1040.

49. Yoshimura S, Ago T, Koga M, Kamouchi M, Kitazono T. Cerebral Small-Vessel Disease in Neuro-Behçet Disease. J Stroke Cerebrovasc Dis 2015;24:e237-e239.

50. Kocer N, Islak C, Siva A, et al. CNS involvement in neuroBehçet syndrome: an MR study. AJNR Am J Neuroradiol 1999;20:1015-1024.

51. Taşolar S, Doğan M, Taşolar H, et al. Evaluation of vertebral artery involvement by Doppler sonography in patients with Behcet disease. J Ultrasound Med 2014;33:811-816.

52. Kose E, Kamisli S, Dogan M, et al. The evaluation of vertebrobasilar artery system in neuro-Behçet and Behçet disease using magnetic resonance angiography. Anat Rec (Hoboken) 2014;297:1302-1305. 